\title{
On Stable and Unstable Growth of Row Structures of Linear-Low-Density Polyethylenes
}

\author{
Moheb Ibrahim ABo el MAATY ${ }^{\dagger}$ \\ J. J. Thomson Physical Laboratory, University of Reading, \\ Reading RG6 6AF, U.K.
}

(Received March 9, 1999)

\begin{abstract}
This paper presents an investigation on the row structures formed by two different kinds of linear-low-density polyethylenes when nucleated on highly oriented polyethylene fibers. The conditions of crystallization have been selected to cover a wide range of temperatures and times in order to study the circumstances giving rise to instability in growth fronts. In both cases the non-crystallizable molecular species which accumulate at the growth front of the row structure during crystallization lower the supercooling causing a progressive decline in the growth rate and an associated coarsening of texture with an increase of interlamellar separation and a wave-like interface. Unstable growth with faster growing protrusions may also develop for Ziegler-Natta catalysed polymer; this does not occur under all crystallization conditions but has only been found when the concentration of the segregated species reaches a sufficient value. This is the case at relatively low crystallization temperatures at which growth is much faster than the diffusion rate of segregated species. At higher temperatures, the growth rate falls increasingly from the beginning of crystallization but stabilizes, together with the coarsening (separation of lamellae) at the last stages. In this steady-state condition the growth rate is depressed to a value equal to the diffusion rate of segregated species.

KEY WORDS Linear-Low-Density Polyethylenes / Cellulation / Row Structures / Segregation / Morphology /
\end{abstract}

Recent papers ${ }^{1-3}$ have reported the first unambiguous observations of uniform crystallization in an undoped polymer becoming morphologically unstable and forming cells. In so doing they have brought clarification of the nature of cellulation and its role in polymer crystallization. As has long been proposed ${ }^{4,5}$ cellulation is a consequence of accumulating segregation of 'impurities' or poorly crystallizable species at the growth front. The new work has focussed attention on the nature of segregants which to be effective must slow the growth rate significantly for instability to set in. So far instability has only been observed for branched polyethylenes, accompanying a continuously declining isothermal growth rate, and poorly isotactic polypropylene. ${ }^{6}$ It has not been observed for the linear polymer nor for highly isotactic polypropylene which continue to grow uniformly indefinitely. ${ }^{7-9}$ The difference between the two cases is that only for the branched polymer-or for poorly tactic polypropylene-are the supercooling for isothermal growth and the growth rate significantly decreased by segregation during crystallization because of a fall in the equilibrium temperature of the local melt.

The concentration of rejected species is maximised for growth on a linear nucleus such as a fiber. This is the system for which instability was first observed, ${ }^{1}$ using a Ziegler-Natta linear-low-density polyethylene (LLDPE), but it occurred in non-steady-state conditions. Progressive changes in habit occurred with increasing radial distance namely a textural coarsening in which lamellae moved further apart on average and began to deviate from the original growth direction with a degree of lamellar thickening followed by nucleation on and growth from the row of faster-growing spherulitic protrusions. On the other hand a survey of the behavior of a range of linear-low-density polyethylenes ${ }^{10}$ showed similar changes but with a smooth transition to a uniform wavy interface without faster growing protrusions both for Ziegler-Natta polymers of low branch content and for all metallocene-catalysed materials to 28 branches/1000 $\mathrm{C}$ atoms. This paper reinforces these earlier observations and goes on to show that faster-growing protrusions do not occur in the highly branched Ziegler-Natta polyethylene at the highest temperatures. It takes the observations of growth rate and coarsening forward quantitatively and in so doing establishes when the steady-state growth is achieved and the nature of this condition.

\section{MATERIALS AND EXPERIMENTAL PROCEDURES}

Two types of polyethylene are used in this work, one referred to as LLDPE, used in previous work, ${ }^{1}$ and the other as polymer A. The latter is a metallocene-catalysed polyethylene, with 1-hexene as the comonomer, i.e., with butyl branches. It has 9.6 branch content per 1000 carbon atoms and average molecular weights $M_{w}=9.65 \times 10^{4}$ and $M_{n}=4.27 \times 10^{4}$. The LLDPE is a Ziegler-type copolymer with 1-hexene, commercially produced by BP Chemicals and is the grade LL0209AA. It has an average branch content of 21.0 butyl branches per 1000 carbon atoms and average molecular weights $M_{w}=1.26 \times 10^{5}$; $M_{n}=3 \times 10^{4}$.

The first experimental step was preparing discs of diameter $7 \mathrm{~mm}$ and thickness $1 \mathrm{~mm}$ by melting the appropriate amount of material at $200^{\circ} \mathrm{C}$ for $10 \mathrm{~min}$ in a Mettler FP82HT hot stage controlled by a Mettler FP90 central processor with nitrogen flow and then quenching in ice-water mixture. Five to seven highly oriented polyethylene fibers, of type Tekmilon (Mitsui)

\footnotetext{
${ }^{\dagger}$ On leave from Physical Science Department, Faculty of Engineering, Mansoura University, Mansoura, Egypt.
} 
manufactured by melt kneading, were parallel stacked on a microscope slide by lightly adhering their ends using a quick drying adhesive. A fiber/material composite was then prepared between the microscope slide and a cover slip by placing a prepared disc on the cover slip on the Kofler hot bench at $130^{\circ} \mathrm{C}$. Onto this the microscope slide with the stacked fibers was carefully placed with light pressure on the already melted disc. The fiber/ material composite with the microscope slide and cover slip was then placed in the Mettler hot stage at $130^{\circ} \mathrm{C}$ for $3 \mathrm{~min}$. The temperature of the hot stage was then dropped to the required crystallization temperature. After the selected time of crystallization, the composite was quenched in ice-water and removed carefully from the slide and cover slip. Observed optically under a Nomarski differential interference microscope, the fibers were found to be embedded around $50 \mu \mathrm{m}$ below the surface of the disc.

All specimens (the fiber/material composites) were etched in an etchant consisting of $2 \% \mathrm{w} / \mathrm{v}$ solution of potassium permanganate dissolved in $5: 2: 2$ by volume concentrated sulphuric acid to $85 \%$ orthophosphoric acid to distilled water. It was found that $4 \mathrm{~h}$ etching time is suitable so that the final plane surface of each specimen passes close to the fiber axis, so that under the Nomarski microscope the etched surfaces showed a width of fibers close to their nominal diameter $(40 \mu \mathrm{m})$. The scanning electron microscope (SEM) was used for quantitative investigations of the dimensions of etched specimens which were coated with gold using an Emscap Sc500 Sputter Coater. Also, some specimens were prepared for transmission electron microscopy, for which a standard two-stage replication method employing cellulose acetate with tantalum-tungsten shadowing and carbon coating has been applied.

\section{RESULTS}

In this work the conditions of crystallization have been chosen to cover a wide range of temperatures and time in order to achieve a more extensive study of habit changes and their instability than before. It has been found that a good quantitative analysis for the behavior of both coarsening and growth rate can be obtained at relatively high crystallization temperatures or in other words, at relatively low supercooling, since there is a long time before the growth of row structures is interrupted due to the nucleation and growth of spherulites, and in addition these conditions do not give rise to the high deviation of the lamellae which would make quantitative analysis difficult. In addition, crystallization at lower temperatures has been undertaken to show the range of instability conditions in our materials and also the validity of the ideas relating to crystallization at relatively higher temperatures.

Figure 1 shows the row structure of polymer $\mathrm{A}$ after $15 \mathrm{~min}$ at $112^{\circ} \mathrm{C}$ crystallization temperature. It is clearly seen that the lamellar growth starts perpendicular to the fiber axis. Close-packed lamellae are nucleated on and share the common $c$ axis of the fibers. The growth near the fiber surface is nearly uniform with thin lamellae which at the outside of the row deviate highly from the original perpendicular growth direction and are more

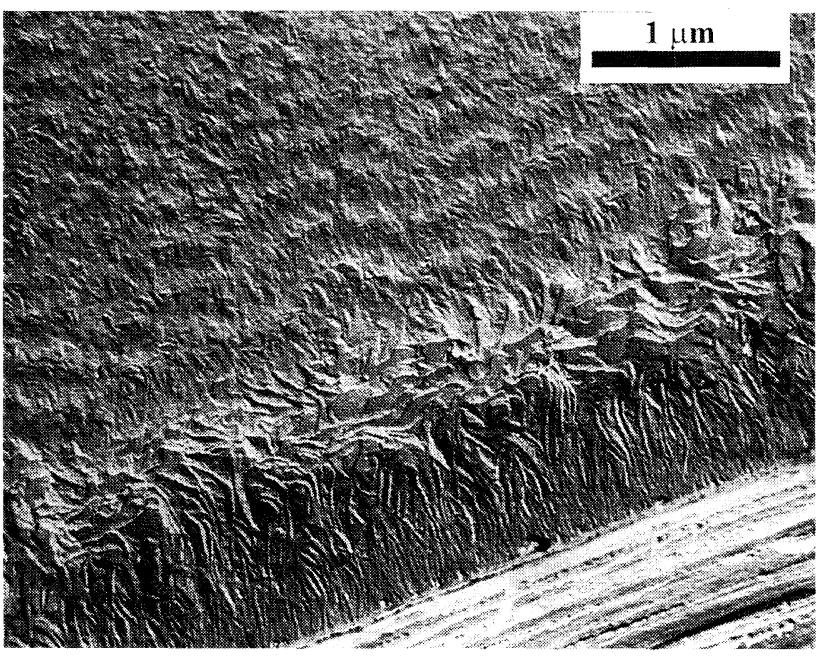

Figure 1. A row structure of Polymer A growing from a Tekmilon fiber ( passing through the lower right corner of the micrograph) and bounded by a quenched matrix. Lamellae in the row structure which originally were close-packed and perpendicular to the fiber have become highly deviated and separated after $15 \mathrm{~min}$ crystallization at $112^{\circ} \mathrm{C}$. Note the wavy bands produced on quenching, indicative of the wavy growth front. Transmission electron micrograph of surface replica of etched surface.

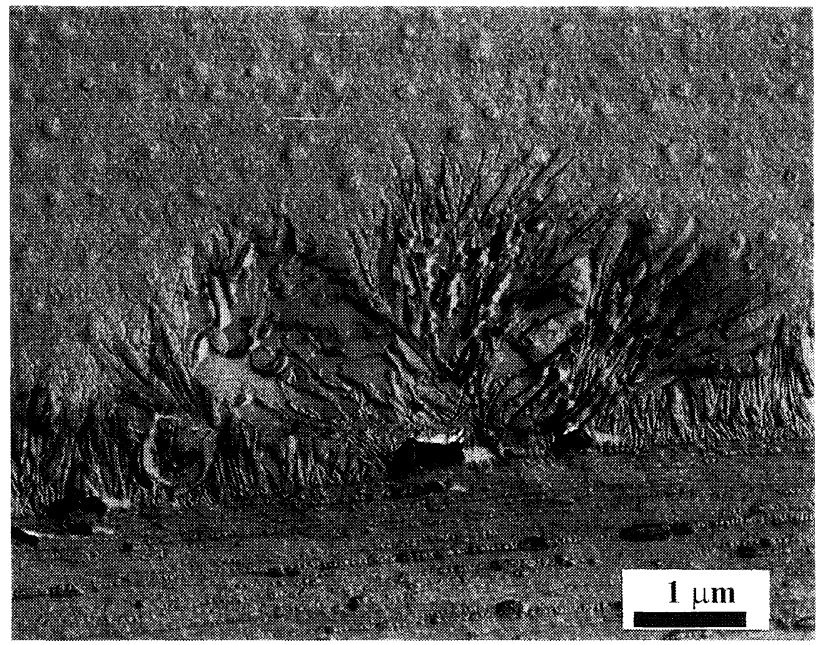

Figure 2. Spherulitic growth nucleating from the row structure of LLDPE, itself nucleated on the Tekmilon fiber crossing the bottom of the photograph, during crystallization at 121 C for $15 \mathrm{~min}$. Transmission electron micrograph of surface replica of etched surface.

separated. At this stage there are fewer, but thicker, lamellae per unit length which change is termed "coarsening"; a quantitative measure of this phenomenon is introduced below. Coarsening can also be seen in the highly deviated lamellae in Figure 1. These stages reproduce phenomena reported previously for LLDPE at the beginning of what those authors" ${ }^{1}$ termed "morphological instability" which in their experiments developed further into spherulitic protuberances, as illustrated in Figure 2.

The difference between the two kinds of polymer, according to our observations here, is that in polymer A, no spherulitic protuberances develop from the row structure nor do spherulites nucleate far from the fibers at $112^{\circ} \mathrm{C}$ while for LLDPE it was found, below $121.5^{\circ} \mathrm{C}$ crystallization temperature, that (faster-growing) spher- 
ulitic protuberances did eventually nucleate sporadically along the row structure as well as independently nucleated spherulites far from the fiber. ${ }^{1}$ In the beginning of crystallization the common interpretation for both materials is that the lamellae are close-packed (Figure 1). Hence there is no room for the segregated (i.e., most-branched) molecules and sequences to reside between the close-packed lamellae. The segregated molecules can then only accumulate on the growth front of row structure. The continuous increase in concentration of the segregated molecules should decrease the local melting point of the material in the melt and so continuously decrease the supercooling and growth rate. This promotes the observed coarsening of texture because if lamellae move further apart, the segregant concentration will be less and the growth rate less depressed.

Coarsening can and does occur without major breakup of the growth front but instability of the growth front with the further development of highly-deviated lamellae and faster growing spherulitic protrusions has only been found to occur when a certain high value of the concentration of suitable segregated species is exceeded, because of insufficient diffusion away. The distinction between the two kinds of LLDPE has been ascribed ${ }^{10}$ to the differing nature of the segregated species or sequences in the two cases related to their respective short-chain branching distributions. Ziegler-Natta polyethylenes, for which high branch content correlates with short molecular length, may well have a greater tendency to segregate whole molecules than do metallocene materials. Spherulitic nucleation from the row structure is observed only in LLDPE and at crystallization temperatures below $122^{\circ} \mathrm{C}$ which is consistent with, but adds to, what was observed before. ${ }^{1,10}$ Figure 2 shows such a spherulitic protrusion from the row structure of LLDPE after crystallization for $15 \mathrm{~min}$ at $121^{\circ} \mathrm{C}$. The coarsening can be seen at the end of both original row and spherulitic structures, consistent with a decrease of supercooling at the end of crystallization in both cases.

In addition to the main features shown in Figure 1, the top of this figure displays a crystallization of the melt during quenching in the form of bands parallel to the fiber. These bands gradually decrease in their width as they move away from the row structure and finally disappear. This is a result of a rapid increase in supercooling during quenching. The quenched bands are not parallel to fiber surface but they have nearly wave-like shape which reflects the actual shape of irregular, cellulating, growth front of the row structure. Cellulated growth fronts were also observed before on polyethylene spherulites ${ }^{2,3}$

It is clear, according to Figures 1 and 2, that although the coarsening is shown in these figures, it is difficult to measure after longer times, especially with high deviation of lamellae in polymer A or with the spherulites nucleated from the row structure in LLDPE. At higher temperatures there is less problem; the row structure of LLDPE, after $60 \mathrm{~min}$ at $122^{\circ} \mathrm{C}$ crystallization temperature, is shown in Figure 3. Although the length of growth is more than $3 \mu \mathrm{m}$, it is still nearly uniform and perpendicular to the fiber. Also, no spherulites are present either growing from or close to the row structure. Down

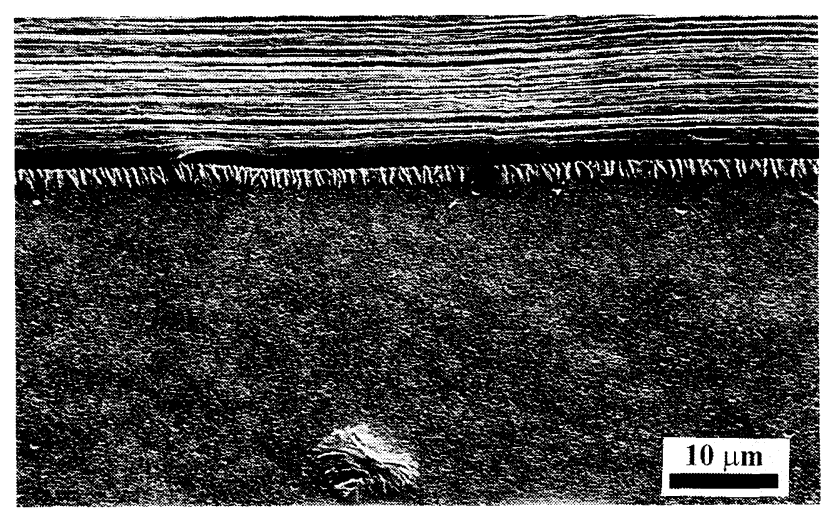

Figure 3. A spherulite with faster growth than the row structure, the latter still uniform and perpendicular to the Tekmilon fiber (at the top of the figure) after $60 \mathrm{~min}$ at $122^{\circ} \mathrm{C}$ crystallization temperature. Scanning electron micrograph for etched surface of LLDPE/fiber composite.

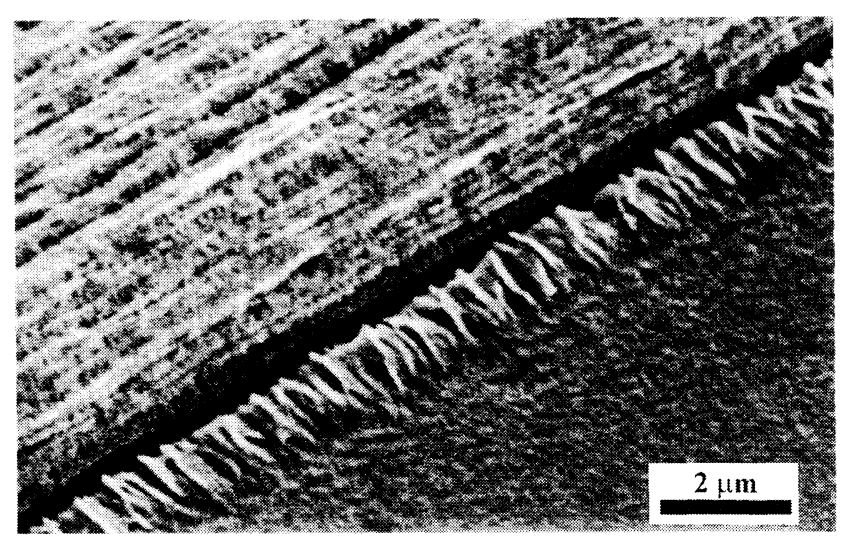

Figure 4. A nearly uniform row structure of polymer A growing from the Tekmilon fiber (in the upper left half of the figure) after $3 \mathrm{~h}$ crystallization at $115^{\circ} \mathrm{C}$. Scanning electron micrograph of etched surface.

and far from the fiber, around $20 \mu \mathrm{m}$ from the row structure in Figure 3, there is a spherulite with radius greater than that of the row structure itself showing that it has the faster growth rate. This means that row structures which grow first can be overtaken by later nucleating spherulites, because these have a more open structure which allows some of non-crystallizable molecules to diffuse into the intervening spaces between their lamellae, as was observed and discussed previously. ${ }^{1}$ Similar behavior of the row structures were observed in polymer A, but at these high temperatures spontaneously nucleated spherulites were not formed. Figure 4 shows a nearly uniform row structure for this material after long time, $3 \mathrm{~h}$, at $115^{\circ} \mathrm{C}$ crystallization temperature.

Figure 5 presents the developing of row structure of LLDPE at $123^{\circ} \mathrm{C}$ crystallization temperature. Lamellae which start to grow as separate individuals adjust and perhaps combine to form units whose identity is emphasized by the limited resolution of the SEM. Of importance is that highly deviated lamellae within, or spherulitic nucleation from, the growing row structure, are not observed at this temperature even after $4.5 \mathrm{~h}$ crystallization time. However, thicker lamellar units were observed after higher crystallization time. This can be seen clearly by following the sequence through Figures 


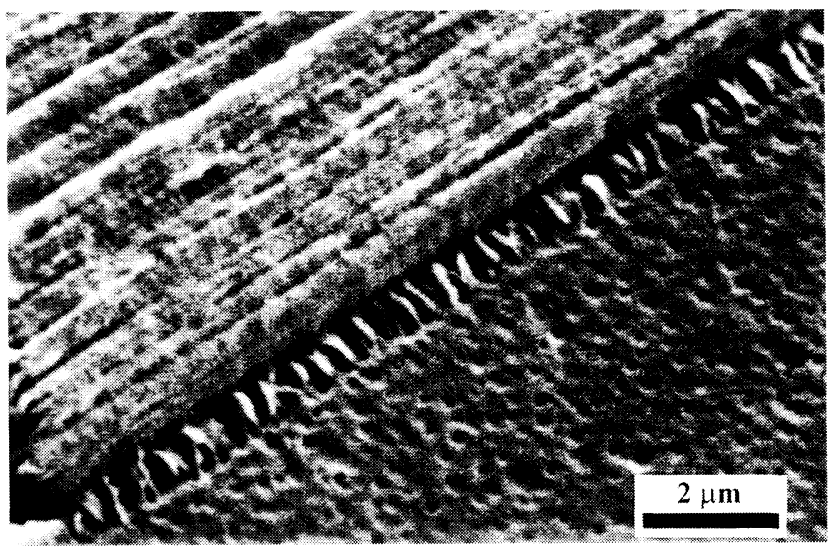

(a)

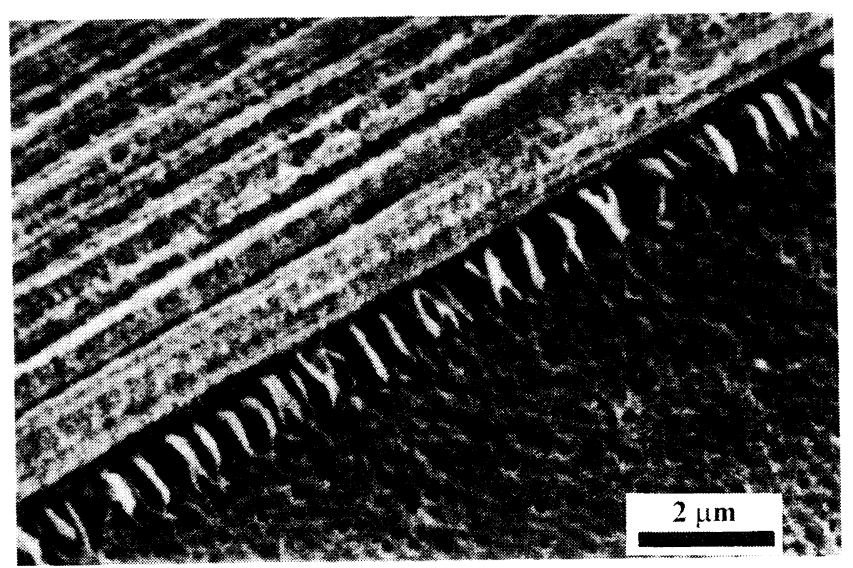

(b)

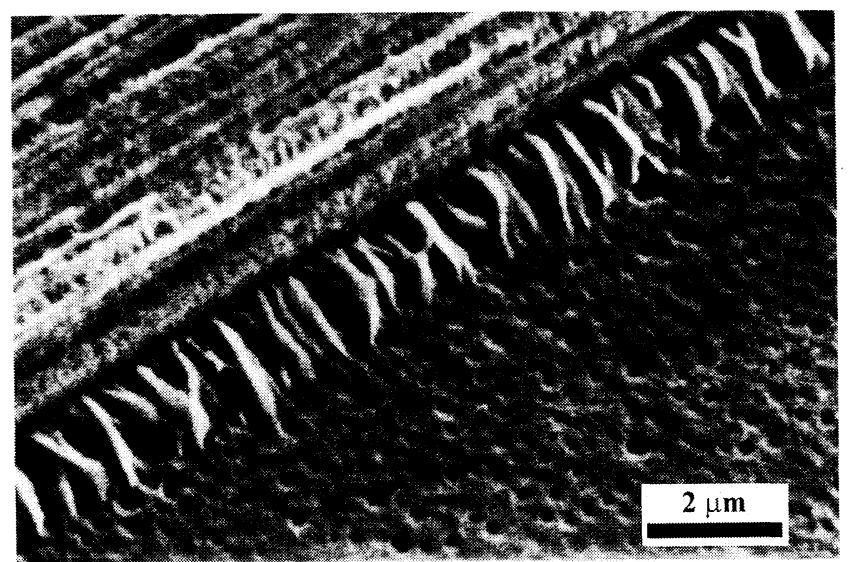

(c)

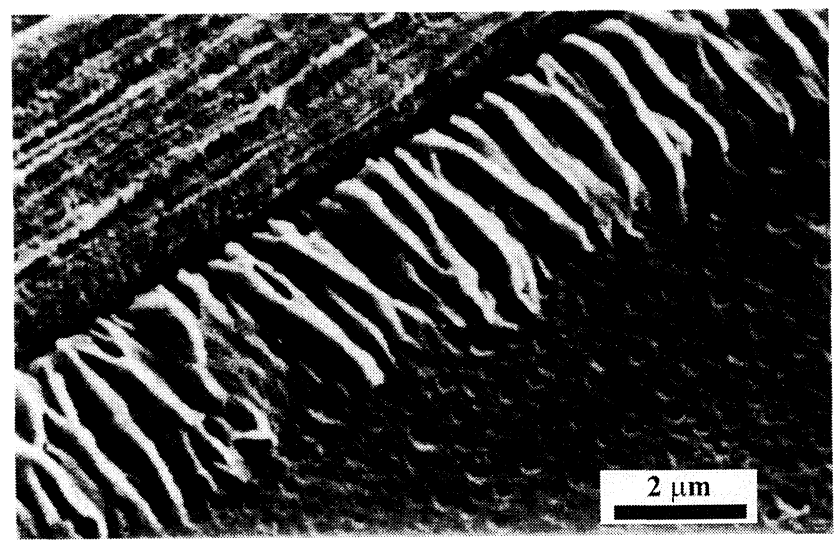

(d)

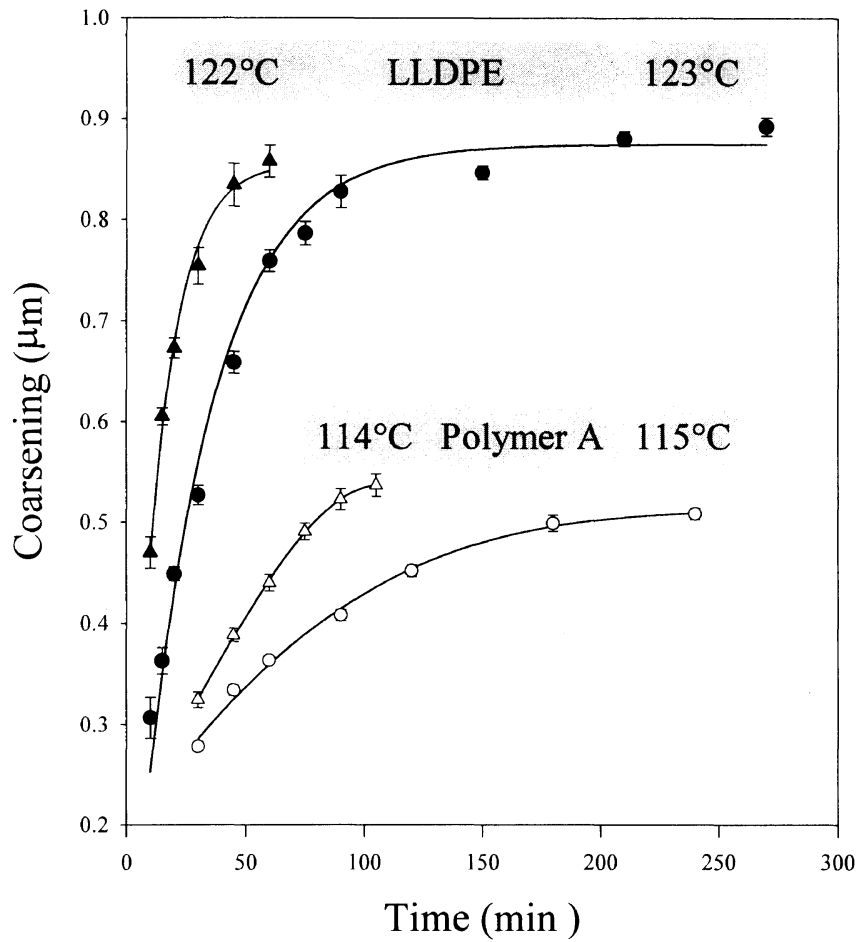

Figure 6. The variation of coarsening of row structures of LLDPE with time at 122 and $123^{\circ} \mathrm{C}$ and of polymer $\mathrm{A}$ at 114 and $115^{\circ} \mathrm{C}$

$5 \mathrm{a}$ to $5 \mathrm{~d}$. It implies that there is a progressive depression of the supercooling and growth rate as the concentration of segregated species increases with time but not sufficiently to reach a value at which spherulitic protuberances are initiated. In other words, the diffusion rate of segregated molecules is certainly less than the growth rate, especially at the earlier stages, but the difference between them still does not give a high enough concentration of segregated species to disrupt the growth front.

Quantitative analysis confirms this idea, as shown in Figure 6 which indicates the coarsening of LLDPE versus the crystallization time at the two crystallization temperatures $122^{\circ} \mathrm{C}$ and $123^{\circ} \mathrm{C}$. Coarsening is indicated in units of $\mu \mathrm{m}$, being the inverse of lamellar density per unit length of row structure, i.e., it is the average separation between the centers of lamellar units, which is the most accurate measure of coarsening. For each temperature and time, the number of lamellar units at the edge of the row was counted per $15 \mu \mathrm{m}$ of row structure (parallel to the fiber axis), to determine the lamellar density per unit length of row structure in $\mu \mathrm{m}^{-1}$, of which the coarsening is the reciprocal value. The coarsening, and also the growth length of row structure for each set of conditions, was then calculated statistically from around 10 different specimens.

The coarsening increases rapidly in the earlier stages but then levels off towards a maximum value and it is seen to saturate to an approximately constant value at the last stages of crystallization. The interpretation of this behavior is that the rapid increase in the coarsening

Figure 5. Row structures of LLDPE crystallized at $123^{\circ} \mathrm{C}$ after (a) $15 \mathrm{~min}$, (b) $30 \mathrm{~min}$, (c) $1 \mathrm{~h}$, and (d) $3.5 \mathrm{~h}$. The nucleating Tekmilon fibers occupy the upper left portion of each photograph. Scanning electron micrographs of etched surfaces. 


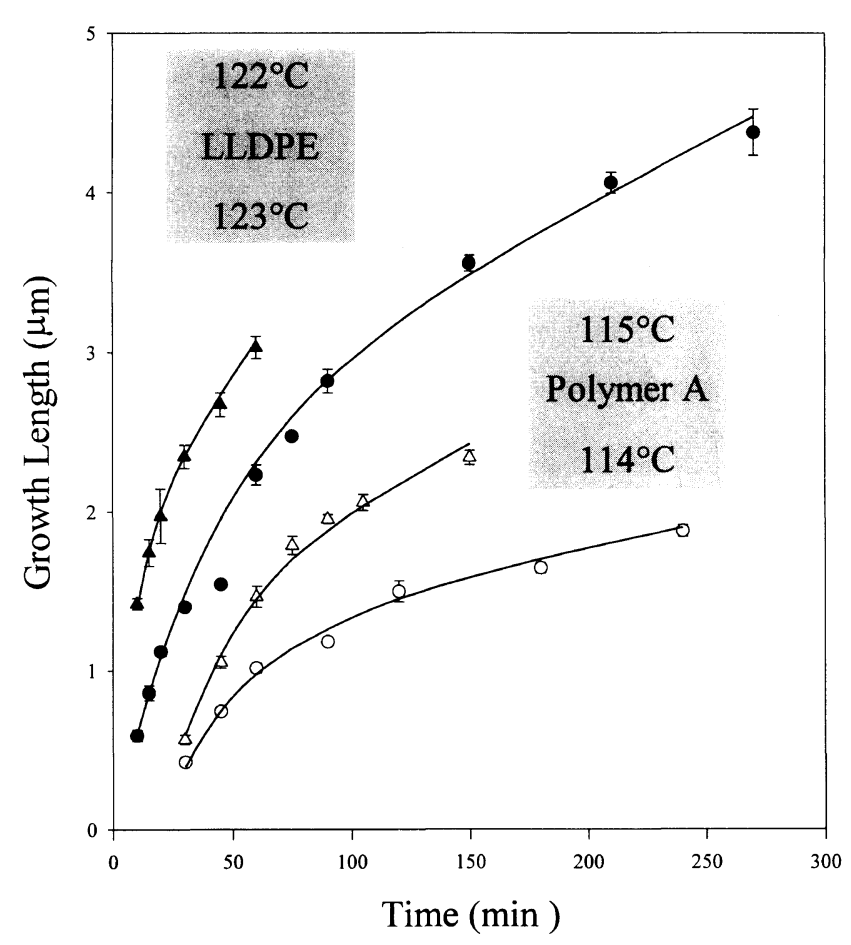

Figure 7. Growth lengths as functions of time for row structures of LLDPE crystallized at 122 and $123^{\circ} \mathrm{C}$ and of polymer $\mathrm{A}$ at 114 and $115^{\circ} \mathrm{C}$.

in the earlier stages is a response by the system to an increased concentration of segregated molecules at the growth front. Greater coarsening reduces their concentration, increases the growth rate and is, therefore, inherently more stable. But, at the later stages, at which there is no measurable increase in coarsening, the effective supercooling becomes approximately constant and there is no significant change in the concentration of segregated species at the lamellar growth faces. Hence the growth rate of row structure should be comparable to the diffusion rate of segregated species. One can expect then that the growth rate at the last stages is constant at nearly constant supercooling and this is what is observed, where Figure 7 represents the growth length versus the time for LLDPE at both $122^{\circ} \mathrm{C}$ and $123^{\circ} \mathrm{C}$ crystallization temperatures. The growth length is seen to be linear with time at the last stages of crystallization.

Similar results were observed for polymer A. Figure 6 shows that the coarsening increases faster at the earlier crystallization time and is nearly constant at the last stages. Figure 8 represents the behavior of growth for row structure of the same material at $114^{\circ} \mathrm{C}$ and $115^{\circ} \mathrm{C}$. Nearly steady-state growth is observed at the later stages, indicating the approximately equal rates for both growth and diffusion.

\section{DISCUSSION}

Changes in the pattern of growth with increasing radius of polyethylene row structures are phenomena associated with crystallization of linear-low-density materials with some branch content; they do not occur for the linear polymer. Increased coarsening with radius is general for branched polyethylenes but the growth of faster-growing, spherulitic, protrusions has not been found at all crystallization conditions, but only in LLDPE and then when the concentration of segregated species reaches a sufficiently high value. This behavior was not seen in polymer A at any crystallization temperature, ${ }^{10}$ although cellulation has occurred in it down to $114^{\circ} \mathrm{C}$. This may indicate a difference in the nature of the segregated molecules or sequences, possibly with longer inter-branch lengths present in those segregated from LLDPE than from Polymer A, a point which requires further investigation.

The possibility of observing habit modifications at relatively lower crystallization temperatures, is represented in Figure 1 for polymer $\mathrm{A}$ and Figure 2 for LLDPE, consistent with the previous work on morphological instability. ${ }^{1,10}$ In both cases the row structure is nucleating on the Tekmilon fiber and starting to grow with transverse lamellae sharing the common $c$ axis of the fibers. The growth near the fiber surface is nearly uniform with thin lamellae which coarsen as they grow far from the fiber and finally are highly deviated from the original perpendicular growth direction. Of the two contributions to coarsening, the increase in separation of lamellae is quite evident at the growth surface while careful observation of lamellae when they are edge on supports the increase of thickness although the prime evidence for thickening is that of Figure 5. Farther out, the growth rate decreases as a result of depressing the supercooling as the concentration of segregated molecular species increases gradually in association with the coarsening discussed above. This change with supercooling is consistent with established trends for polyethylene crystallization ${ }^{11}$ and continues until the level of impurities reaches enough to perturb the growth surface.

The banding of crystal texture depends strictly on the supercooling; narrower bands can be found at higher supercooling. ${ }^{12,13}$ This is observed in the top of Figure 1 , where the size of bands decreases gradually as they move away from the row structure, as a result of rapid increasing of supercooling during quenching. The original row structure in this case is not banded, although banded row structures have been seen in linear polyethylene from the Leeds compaction process, ${ }^{14}$ provided the cooling rate is sufficiently high, and in LLDPE. ${ }^{1,10}$ The non-banded case indicates a crystallization at lower supercooling.

Spherulitic nucleation from the row structure is observed only in LLDPE at crystallization temperatures below $122^{\circ} \mathrm{C}$, consistent with what was found in the previous work, ${ }^{1,10}$ in which it was found that such spherulitic nucleation falls as crystallization temperature rises. So, at higher temperatures, it is expected to find row structures free from spherulitic nucleation. This is what is observed in LLDPE, in which at $122^{\circ} \mathrm{C}$ and $123^{\circ} \mathrm{C}$ there is no nucleation at around $20 \mu \mathrm{m}$ around the fiber, but farther out, there are independently nucleated spherulites and their growth rate is faster than for the original row structure (see Figure 3). In contrast to this, spherulitic protrusions from row structures were found at $121^{\circ} \mathrm{C}$ (see Figure 2) and below. It has not occurred at the relatively higher temperatures even after very long crystallization time because, although 
the supercooling is depressed as a result of increasing the concentration of segregated species, but this does not increase sufficiently to interrupt the growth surface. This has been reflected in this work through the behavior of both coarsening and growth rate both of which are good indicators because they reflect the change in supercooling during crystallization.

At the higher temperatures, the texture coarsens very rapidly at the beginning of crystallization and becomes nearly constant in the last stages (Figures 6 and 8). The progressive nature of the depression of supercooling, from the very first stages, can be followed in the variation of coarsening along the Figures $5 \mathrm{a}$ to $5 \mathrm{~d}$. In these figures, there can also be seen the slightly curved lamellae which are indicative of crystallization at lower supercooling. ${ }^{15}$ At the last stages of crystallization at higher temperatures, there is no significant change in coarsening, being now the case at which the growth rate reaches to value close to the diffusion rate of segregated molecules at this temperatures, i.e., at fixed species concentration.

The behavior of growth rate is in accordance with the morphological observations. At the beginning of crystallization, the rate declines as a result of decreasing supercooling (Figures 7 and 9). This continues until the growth rate becomes close to the diffusion rate of segregated molecules, the case at which a fixed species concentration is achieved. This is in fact achieved at the later stages of crystallization as represented by the straight end portions of curves of Figures 7 and 9. The steady state or linear growth is then observed at the fixed supercooling.

\section{CONCLUSIONS}

The main conclusions of this work can be written as follows:

1. Radial changes in habit for row structures of polyethylene is a phenomenon associated with the growth of low density polyethylene which has a certain amount of branch content, in which the non-crystallizable, more-branched molecules or sequences accumulate at the growth front during crystallization and then significantly lower the supercooling.

2. Unstable growth fronts with spherulitic protrusions occur just in LLDPE and then only if the noncrystallizable species concentration reaches a high enough value. It is accordingly more common at lower crystallization temperatures when there is a higher difference between the growth rate and diffusion of segregated species and disappears at high temperatures. Its absence from metallocene materials indicates a dependence on the nature of the segregated species presumably related to the short chain branching distributions.

3. The microstructure (coarsening) adjusts with the growth rate as both accommodate to the concentrations of segregated species. At higher crystallization temperatures, the growth rate declines initially but at the later stages, linear growth rate and coarsening were both observed to stabilize and become constant. In steadystate conditions the growth rate equals the diffusion rate of segregated species.

Acknowledgments. This work is a part of a research program supported by NEDO, the New Energy and Industrial Technology Development Organization of Japan. The author would like to thank both Prof. D. C. Bassett and R. H. Olley for useful advice and help.

\section{REFERENCES}

1. J. J. Janimak and D. C. Bassett, Polymer, 40, 459 (1999).

2. M. I. Abo el Maaty, I. L. Hosier, and D. C. Bassett, Macromolecules, 31, 153 (1998).

3. M. I. Abo el Maaty, D. C. Bassett, R. H. Olley, and P. Jääskeläinen, Macromolecules, 33, 7800 (1998).

4. H. D. Keith and F. J., Padden, J. Appl. Phys., 34, 2409 (1963).

5. S. Balijepalli and J. M. Schultz, Macromolecules, 29, 2095 (1996).

6. H. M. White, Ph. D Thesis, University of Reading (1995).

7. A. J. Pennings, Polym. Sci. Symp., 59, 55 (1977).

8. B. Maxwell, J. Polym. Sci. C, 9, 43 (1965).

9. J. Varga and J. Karger-Kocsis, J. Polym. Sci., B, Polym. Phys. Ed., 34, 657 (1996).

10. I. L. Hosier and D. C. Bassett, Polym. J., 31, 772 (1999).

11. J. D. Hoffman, L. J. Frolen, G. S. Ross, and J. I. Lauritzen, J. Res. Nat. Bur. Stand. A, 79, 671 (1975).

12. A. Keller, in "Growth and Perfection of Crystals," R. H. Doremus, B. W. Roberts, and D. Turnbull, Ed., WileyInterscience, Chichester, 1958, p 499.

13. P. J. Phillips, in "Bulk Crystal Growth," D. T. J. Hurle, Ed., North Holland, Amsterdam, 1993, p 1171.

14. M. A. Kabeel, D. C. Bassett, R. H. Olley, P. J. Hine, and I. M. Ward, J. Mater. Sci., 30, 601

15. D. C. Bassett and A. M. Hodge, Proc. R. Soc. London, A, 377, 25; 39 (1981) 